\title{
GRADIENT ESTIMATES FOR MEAN CURVATURE FLOW WITH NEUMANN BOUNDARY CONDITIONS
}

\author{
MASASHI MIZUNO AND KEISUKE TAKASAO
}

\begin{abstract}
We study the mean curvature flow of graphs both with Neumann boundary conditions and transport terms. We derive boundary gradient estimates for the mean curvature flow. As an application, the existence of the mean curvature flow of graphs is presented. A key argument is a boundary monotonicity formula of a Huisken type derived using reflected backward heat kernels. Furthermore, we provide regularity conditions for the transport terms.
\end{abstract}

\section{INTRODUCTION}

We consider the mean curvature flow of graphs with transport terms and Neumann boundary conditions:

$$
\left\{\begin{aligned}
\frac{\partial_{t} u}{\sqrt{1+|d u|^{2}}} & =\operatorname{div}\left(\frac{d u}{\sqrt{1+|d u|^{2}}}\right)+\boldsymbol{f}(x, u, t) \cdot \boldsymbol{n} & & x \in \Omega, t>0, \\
\left.d u \cdot \nu\right|_{\partial \Omega} & =0, & & t>0, \\
u(x, 0) & =u_{0}(x), & & x \in \Omega,
\end{aligned}\right.
$$

where $\Omega \subset \mathbb{R}^{n}$ is a bounded domain with a smooth boundary, $\nu$ is an outer unit normal vector on $\partial \Omega, u=u(x, t): \Omega \times[0, \infty) \rightarrow \mathbb{R}$ is an unknown function, $d u:=\left(\partial_{x_{1}} u, \ldots, \partial_{x_{n}} u\right)$ is the tangential gradient of $u, u_{0}=u_{0}(x): \Omega \rightarrow \mathbb{R}$ is given initial data, $\boldsymbol{f}: \Omega \times \mathbb{R} \times$ $[0, \infty) \rightarrow \mathbb{R}^{n+1}$ is a given transport term, and $\boldsymbol{n}=\frac{1}{\sqrt{1+|d u|^{2}}}(-d u, 1)$. For a solution $u$ of (1.1) and $t>0$, the graph of $u(x, t)$, which is

$$
\Gamma_{t}:=\{(x, u(x, t)): x \in \Omega\},
$$

satisfies the mean curvature flow with the transport term, which is subjected to right angle boundary conditions given by

$$
\left\{\begin{array}{lr}
\boldsymbol{V}=\boldsymbol{H}+(\boldsymbol{f} \cdot \boldsymbol{n}) \boldsymbol{n}, & \text { on } \Gamma_{t}, t>0, \\
\Gamma_{t} \perp \partial(\Omega \times \mathbb{R}), & t>0,
\end{array}\right.
$$

where $\boldsymbol{n}:=\frac{1}{\sqrt{1+|d u|^{2}}}(-d u, 1)$ is the unit normal vector of $\Gamma_{t}, \boldsymbol{V}:=\frac{\partial_{t} u}{\sqrt{1+|d u|^{2}}} \boldsymbol{n}$ is the normal velocity vector of $\Gamma_{t}$, and $\boldsymbol{H}:=\operatorname{div}\left(\frac{d u}{\sqrt{1+|d u|^{2}}}\right) \boldsymbol{n}$ is the mean curvature vector of $\Gamma_{t}$ (see Figure 10. It is interesting to derive the regularity criterion of the transport term

2000 Mathematics Subject Classification. Primary 35K93, Secondary 53C44,35B65.

Key words and phrases. Mean curvature flow, boundary gradient estimates, boundary monotonicity formula.

This work was supported by JSPS KAKENHI Grant Numbers 25800084, 25247008. 


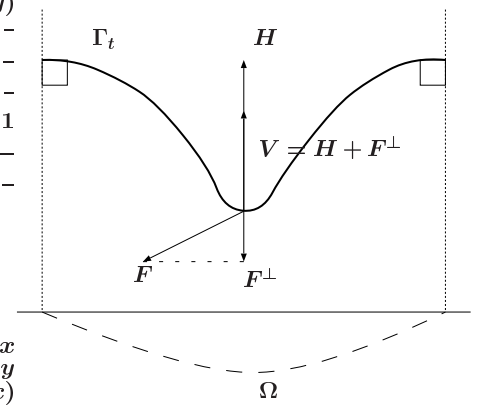

Figure 1. Mean curvature flow with the transport $\boldsymbol{F}$ and with the Neumann boundary condition.

to obtain the classical solution of (1.1). Liu-Sato-Tonegawa 18] studied the following incompressible and viscous non-Newtonian two-phase fluid flow:

$$
\begin{cases}\frac{\partial \boldsymbol{f}}{\partial t}+\boldsymbol{f} \cdot \nabla \boldsymbol{f}=\operatorname{div}\left(T^{ \pm}(\boldsymbol{f}, \Pi)\right), \operatorname{div} \boldsymbol{f}=0, & \text { on } \Omega_{t}^{ \pm}, t>0 \\ \boldsymbol{n} \cdot\left(T^{+}(\boldsymbol{f}, \Pi)-T^{-}(\boldsymbol{f}, \Pi)\right)=\boldsymbol{H}, & \text { on } \Gamma_{t}, t>0 \\ \boldsymbol{V}=\boldsymbol{H}+(\boldsymbol{f} \cdot \boldsymbol{n}) \boldsymbol{n}, & \text { on } \Gamma_{t}, t>0\end{cases}
$$

where $\Omega_{t}^{+} \cup \Omega_{t}^{-} \cup \Gamma_{t}=\mathbb{T}^{n+1}=(\mathbb{R} / \mathbb{Z})^{n+1}, \boldsymbol{f}$ is the velocity vector of the fluids, $T^{ \pm}$is the stress tensor of the fluids, and $\Pi$ is the pressure of the fluids. The physical background of (1.4) was studied by Liu-Walkington [19]. The phase boundary $\Gamma_{t}$ moves by the fluid flow and its mean curvature. In (1.3), the transport term is corresponding to the fluid velocity of (1.4). Since the regularity of non-Newtonian fluid flow is still difficult problems, it is important to study regularity conditions to obtain the classical solution of (1.1).

To study the behavior of $\Gamma_{t}$, we need to investigate $v:=\sqrt{1+|d u|^{2}}$, which is the volume element of $\Gamma_{t}$. Thus, it is important to derive gradient estimates for (1.1). Interior gradient estimates for (1.1) under $\boldsymbol{f} \equiv 0$ were studied by Ecker-Huisken [8] when the initial surface is $C^{1}$, and by Colding-Minicozzi II [4] when $u_{0}$ is bounded. It is difficult to apply their arguments to (1.1) under non-smooth transport terms because their arguments essentially use the comparison arguments.

Ecker-Huisken [7] also derived the interior gradient estimates for (1.1) under $\boldsymbol{f} \equiv 0$. In their arguments, monotonicity formula is crucial to show the gradient estimates. Takasao [25] studied the interior gradient estimates for (1.1) when $u_{0}$ is $C^{1}$ and the transport $\boldsymbol{f}$ is bounded in time and space variables. An essential part of Takasao's proof is to derive the monotonicity formula of the Huisken type with the bounded transport terms.

Huisken [12] studied (1.1) with the Neumann boundary condition and without the transport $\boldsymbol{f}$. He showed the existence of a classical solution of (1.1) under $\boldsymbol{f} \equiv 0$. To show the existence of the solution, it is important to derive up-to-boundary a priori gradient estimates of (1.1). Huisken showed the gradient estimates when the initial data $u_{0}$ is $C^{2, \alpha}$ up to boundary and $\partial \Omega$ is of class $C^{2, \alpha}$ via the Schauder estimates. Stahl [22] also considered the gradient estimates of (1.1) without the transport and obtained some blow-up criterion of the classical solution of (1.1) under $f \equiv 0$. Up-to-boundary a priori gradient estimates of the mean curvature flow with the Neumann boundary condition are studied by many researchers and we mention [2, 3, 9, 10, 16, 21, 22, 23, 24, 27, 28, 29, Our 
arguments are similar to Ecker's or Takasao's work [5, 25]. In our setting, we need to derive a boundary monotonicity formula for (1.1). From this point, Buckland [3] obtained the boundary monotonicity formula for (1.1) under $\boldsymbol{f} \equiv 0$. Takasao derived the monotonicity formula for (1.1) with transport terms in [25] but the condition was not optimal. On the other hand, reasonable conditions for the transport terms for the regularity of weak mean curvature flow were obtained in [15, 26]. In this paper, we obtain a priori gradient estimates with reasonable conditions for the transport terms.

Our problem (1.1) imposes Neumann boundary conditions; thus, up-to-the-boundary gradient estimates are also important. Mizuno and Tonegawa [20] studied weak mean curvature flow with Neumann boundary conditions via phase field methods. To study boundary behavior, it was important to derive an $\varepsilon$-diffused boundary monotonicity formula of a Huisken type via reflected backward heat kernels (cf. [13], [14]). Thus, it is also important to derive the boundary monotonicity formula for (1.1) and determine the optimal regularity condition for the transport terms. In this paper, we derive the boundary monotonicity formula for (1.1) and as an application, we derive a priori boundary gradient estimates and prove the existence of a classical solution of (1.1).

This paper is organized as follows. In section 2, we present basic notation and the main results. In section 3, we derive the boundary monotonicity formula for (1.1). In section 4 , we derive the up-to-boundary gradient estimates for (1.1) and some integral estimates for the transport terms. In section 5, we prove the existence of the classical solution of (1.1). In section 6, we discuss some optimality for the transport terms to obtain the gradient estimates for (1.1).

\section{Preliminaries AND MAin RESUlts}

2.1. Notation. Let $\boldsymbol{\nu}$ be an outer unit normal vector on $\partial(\Omega \times \mathbb{R}) ; \boldsymbol{\nu}=(\nu, 0)$. For $n$-dimensional symmetric matrices $A$ and $B$, define the inner product $A$ : $B$ as $A$ : $B=\operatorname{tr}(A B)$. Set $Q_{T}:=\Omega \times(0, T)$ and $Q_{T}^{\varepsilon}:=\Omega \times(\varepsilon, T)$ for $0<\varepsilon<T$. Let $d$ and $D$ be the gradients on $\Omega$ and $\Omega \times \mathbb{R}$, respectively. Let $D_{\Gamma_{t}}$ and $\Delta_{\Gamma_{t}}$ be the covariant differentiation and Laplace-Beltrami operator on $\Gamma_{t}$, respectively. For a solution $u$ of (1.1), let $h:=-\operatorname{div}\left(\frac{d u}{\sqrt{1+|d u|^{2}}}\right), v:=\sqrt{1+|d u|^{2}}$. Then, equation (1.1) becomes

$$
\partial_{t} u=-v h+(\boldsymbol{f} \cdot \boldsymbol{n}) v .
$$

2.2. Main results. Let $T_{0}>0$ be fixed. We impose a regularity assumption on the transport term such that for $p, q \geq 1$ satisfying $\frac{n}{p}+\frac{2}{q}<1$,

$$
\|\boldsymbol{f}\|_{L_{t}^{q} L_{x}^{p}\left(0, T_{0}, \Gamma_{t}\right)}:=\left(\int_{0}^{T_{0}}\left(\int_{\Gamma_{t}}|\boldsymbol{f}(X, t)|^{p} d \mathscr{H}^{n}\right)^{\frac{q}{p}} d t\right)^{\frac{1}{q}}<\infty .
$$

Remark 2.1. Using the Meyer-Ziemer inequality (cf. [30, p. 266, Theorem 5.12.4]),

$$
\int_{\Gamma_{t}}|\boldsymbol{f}(X, t)|^{p} d \mathscr{H}^{n} \leq C\|\boldsymbol{f}(\cdot, t)\|_{W^{1, p}(\Omega \times \mathbb{R})}^{p}
$$

hence, our assumption (2.2) is fulfilled if $\boldsymbol{f} \in L^{q}\left(\left[0, T_{0}\right]: W^{1, p}(\Omega \times \mathbb{R})\right)$.

First we derive a priori gradient estimates for (1.1). 
Theorem 2.2 (A priori estimates for the gradient). Let $u$ be a classical solution of (1.1) on $\Omega \times\left(0, T_{0}\right)$. Assume that $\Omega$ is convex, $u_{0} \in W^{1, \infty}(\Omega)$, and the transport term $\boldsymbol{f}$ satisfies (2.2). Then there exists $T>0$ depending only on $n, p, q, T_{0},\left\|d u_{0}\right\|_{\infty},\|\boldsymbol{f}\|_{L_{t}^{q} L_{x}^{p}\left(0, T_{0}, \Gamma_{t}\right)}$, and $\Omega$ such that

$$
\sup _{0<t<T, x \in \bar{\Omega}} \sqrt{1+|d u(x, t)|^{2}} \leq 4\left(1+\left\|d u_{0}\right\|_{\infty}^{2}\right) .
$$

The regularity assumption (2.2) is reasonable from blow-up arguments. Indeed, using the scale transform

$$
x=\lambda y, \quad t=\lambda^{2} s, \quad w(y, s)=\frac{1}{\lambda} u(x, t),
$$

we obtain

$$
\left\{\begin{aligned}
\frac{\partial_{s} w}{\sqrt{1+|d w|^{2}}} & =\operatorname{div}\left(\frac{d w}{\sqrt{1+|d w|^{2}}}\right)+\lambda \boldsymbol{f}\left(\lambda y, \lambda w, \lambda^{2} s\right) \cdot \frac{(-d w, 1)}{\sqrt{1+|d w|^{2}}} \\
d w & =d u
\end{aligned}\right.
$$

Then

$$
\left\|\lambda \boldsymbol{f}\left(\lambda y, \lambda w, \lambda^{2} s\right)\right\|_{L_{s}^{q} L_{y}^{p}}=\lambda^{1-\frac{n}{p}-\frac{2}{q}}\|\boldsymbol{f}\|_{L_{t}^{q} L_{x}^{p}}
$$

and $\left\|\lambda \boldsymbol{f}\left(\lambda y, \lambda w, \lambda^{2} s\right)\right\|_{L_{s}^{q} L_{y}^{p}} \rightarrow 0$ as $\lambda \rightarrow 0$ if (2.2) is fulfilled; that is, the transport is a small perturbation for blow-up arguments. Note that the regularity assumption (2.2) is the same as the assumption for the parabolic Allard's regularity theory developed by Kasai-Tonegawa [15, 26]. Furthermore, our results include results from the study by Takasao 25] because our argument also applies to interior gradient estimates. We further explain in Section 6 if (2.2) is fulfilled for $\frac{n}{p}+\frac{2}{q}>1$, then there is a solution of (1.1) such that the gradient of the solution is unbounded.

From the regularity estimate (2.3), the graph $\Gamma_{t}$ subjected to (1.2) is a $C^{1}$-Riemannian manifold up to the boundary. Furthermore, the graph $\Gamma_{t}$ is perpendicular to $\partial \Omega \times$ $\mathbb{R}$, which is the boundary of a cylinder $\Omega \times \mathbb{R}$. In terms of partial differential equations, Theorem 2.2 can be regarded as an up-to-the-boundary parabolic smoothing effect for $\partial_{t} u-\sqrt{1+|d u|^{2}} \operatorname{div}\left(\frac{d u}{\sqrt{1+|d u|^{2}}}\right)$. The non-divergence elliptic differential operator $-\sqrt{1+|d u|^{2}} \operatorname{div}\left(\frac{d u}{\sqrt{1+|d u|^{2}}}\right)$ is degenerate hence regularity for solutions of (1.1) is not clear. When the gradient of solutions is bounded, then the Schauder estimates for (1.1) is applicable thus the higher regularity of solutions and the existence of a solution of (1.1) can be deduced. Theorem 2.2 also can be regarded as a parabolic smoothing effect for the mean curvature operator. To summarize, (2.3) determines how we obtain regularity of the mean curvature flow.

To obtain the gradient estimates via the comparison arguments, the boundedness of $\|D \boldsymbol{f}\|_{\infty}$ is needed. On the other hand, to obtain the gradient estimates via the monotonicity formula of the Huisken type, the boundedness of $\|D \boldsymbol{f}\|_{\infty}$ is not needed. Note that the idea using the weighted monotonicity formula is called the noncompact maximum principle [5, Proposition 4.27]. To show the up-to-boundary monotonicity formula, we introduce reflected backward heat kernels to compute the boundary integrals and derive integral estimates for the transport terms under the assumption (2.2). 
Next, we demonstrate the existence of a classical solution of (1.1). We assume parabolic Hölder continuity for $\boldsymbol{f}$; that is, there is $\alpha \in(0,1]$ such that

$$
K:=\sup _{(X, t),(Y, s) \in(\Omega \times \mathbb{R}) \times\left(0, T_{0}\right)} \frac{|\boldsymbol{f}(X, t)-\boldsymbol{f}(Y, s)|}{|X-Y|^{\alpha}+|t-s|^{\alpha / 2}}<\infty .
$$

Theorem 2.3 (Existence of a classical solution). Assume that $\Omega$ is convex, $u_{0} \in W^{1, \infty}(\Omega)$ with $d u_{0} \cdot \nu \equiv 0$ on $\partial \Omega$ and the transport term $\boldsymbol{f}$ satisfies (2.4) with some $\alpha \in(0,1]$. Then, there exists a time local unique solution $u \in C\left(\overline{Q_{T}}\right) \cap C^{2, \alpha}\left(Q_{T}^{\varepsilon}\right)$ for all $\varepsilon>0$ of (1.1) with $u(0)=u_{0}$ for some $0<T<T_{0}$. Furthermore, for any $\varepsilon>0$ there exists $C_{1}>0$ depending only on $n, \alpha, L, K$ such that

$$
\|u\|_{C^{2, \alpha}\left(Q_{T}^{\varepsilon}\right)} \leq C_{1} .
$$

Theorem 2.3 is deduced from the Leray-Schauder fixed point theorem for the linearized problem of (1.1). Theorem 2.2 is employed as a priori gradient estimates for the LeraySchauder fixed point theorem. As a result of the gradient bounds, the linearized problem of (1.1) can be computed in the same class as the uniformly elliptic operator; hence, we can derive the Schauder estimates for (1.1) and apply the Leray-Schauder fixed point theorem.

\section{Monotonicity of the Metric}

Our first task is to establish the up-to-the-boundary monotonicity formula of the Huisken type.

Lemma 3.1. Let $u$ be a classical solution of (1.1) and $v:=\sqrt{1+|d u|^{2}}$. Then

$$
\partial_{t} v-\Delta_{\Gamma_{t}} v-\left(\frac{d u}{v} \cdot d v\right) \frac{\partial_{t} u}{v}=-\left|A_{t}\right|^{2} v-\frac{2\left|D_{\Gamma_{t}} v\right|^{2}}{v}+d u \cdot d(\boldsymbol{f} \cdot \boldsymbol{n}),
$$

where $D_{\Gamma_{t}}, \Delta_{\Gamma_{t}}$ and $\left|A_{t}\right|$ denote covariant differentiation in $\Gamma_{t}$, Laplace-Beltrami operator, norm of second fundamental form of $\Gamma_{t}$ respectively.

Proof. According to Ecker-Huisken [6],

$$
-\Delta_{\Gamma_{t}} v+\left|A_{t}\right|^{2} v+\frac{2\left|D_{\Gamma_{t}} v\right|^{2}}{v}-v^{2}\left(D_{\Gamma_{t}} h \cdot \boldsymbol{e}_{n+1}\right)=0,
$$

where $\boldsymbol{e}_{n+1}=(0, \ldots, 0,1)$. Because

$$
\begin{aligned}
v^{2}\left(D_{\Gamma_{t}} h \cdot \boldsymbol{e}_{n+1}\right) & =v^{2}\left(D h \cdot \boldsymbol{e}_{n+1}-(D h \cdot \boldsymbol{n})\left(\boldsymbol{n} \cdot \boldsymbol{e}_{n+1}\right)\right) \\
& =d h \cdot d u \\
& =-d\left(\frac{\partial_{t} u}{v}\right) \cdot d u+d(\boldsymbol{f} \cdot \boldsymbol{n}) \cdot d u \quad(\because(1.1)) \\
& =-\partial_{t} v+\left(\frac{d u}{v} \cdot d v\right) \frac{\partial_{t} u}{v}+d(\boldsymbol{f} \cdot \boldsymbol{n}) \cdot d u,
\end{aligned}
$$

we obtain (3.1).

Let

$$
R:=\frac{1}{\| \text { principal curvature of } \partial \Omega \|_{L^{\infty}(\partial \Omega)}}
$$




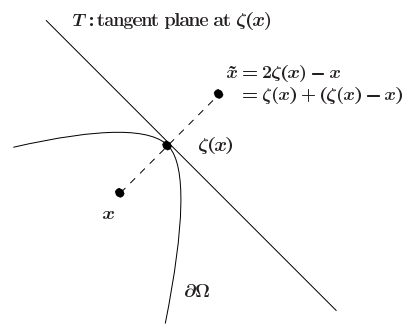

FiguRE 2. The reflection point of $x \in \Omega \cap N_{r}$ with respect to $\partial \Omega$ is denoted by $\tilde{x}$.

Because $\partial \Omega$ is smooth and compact, $0<R<\infty$. For $r<R$, let $N_{r}$ denote the interior tubular neighborhood of $\partial \Omega$;

$$
N_{r}:=\{x \in \Omega: \operatorname{dist}(x, \partial \Omega)<r\} .
$$

For $x \in N_{r}$, there uniquely exists $\zeta(x) \in \partial \Omega$ such that $\operatorname{dist}(x, \partial \Omega)=|x-\zeta(x)|$. Thus, define the reflection point $\tilde{x}$ with respect to $\partial \Omega$ as $\tilde{x}=2 \zeta(x)-x$ (see Figure 2). We fix a radially symmetric cut-off function $\eta_{R}=\eta_{R}(|X|) \in C^{\infty}\left(\mathbb{R}^{n+1}\right)$ such that

$$
0 \leq \eta_{R} \leq 1, \quad \eta_{R}^{\prime} \leq 0, \quad \text { spt } \eta_{R} \subset B_{R / 8}, \quad \eta_{R}=1 \text { on } B_{R / 16} .
$$

For $0<t<s<T_{0}$ and $X=\left(x, x_{n+1}\right), Y=\left(y, y_{n+1}\right) \in N_{R} \times \mathbb{R}$, we define the $n$-dimensional backward heat kernel $\rho_{(Y, s)}(X, t)$ and reflected backward heat kernel $\tilde{\rho}_{(Y, s)}(X, t)$ as

$$
\begin{aligned}
& \rho_{(Y, s)}(X, t):=\frac{1}{(4 \pi(s-t))^{\frac{n}{2}}} \exp \left(-\frac{|X-Y|^{2}}{4(s-t)}\right), \\
& \tilde{\rho}_{(Y, s)}(X, t):=\frac{1}{(4 \pi(s-t))^{\frac{n}{2}}} \exp \left(-\frac{|\tilde{X}-Y|^{2}}{4(s-t)}\right),
\end{aligned}
$$

where $\tilde{X}=\left(\tilde{x}, x_{n+1}\right)$. For fixed $0<t<s$ and $X, Y \in N_{R} \times \mathbb{R}$, we define a truncated version of $\rho$ and $\tilde{\rho}$ as

$$
\begin{aligned}
& \rho_{1}=\rho_{1}(X, t):=\eta_{R}(X-Y) \rho_{(Y, s)}(X, t), \\
& \rho_{2}=\rho_{2}(X, t):=\eta_{R}(\tilde{X}-Y) \tilde{\rho}_{(Y, s)}(X, t) .
\end{aligned}
$$

To derive Huisken's monotonicity formula,

$$
\frac{(\boldsymbol{w} \cdot D \rho)^{2}}{\rho}+\left((I-\boldsymbol{w} \otimes \boldsymbol{w}): D^{2} \rho\right)+\partial_{t} \rho=0
$$

is the crucial identity, where $\rho=\rho_{(Y, s)}(X, t), \boldsymbol{w} \in \mathbb{R}^{n+1}$ is any unit vector, $I$ is the identity matrix, $\boldsymbol{w} \otimes \boldsymbol{w}$ is tensor product, and $(I-\boldsymbol{w} \otimes \boldsymbol{w}): D^{2} \rho$ is $\operatorname{tr}\left((I-\boldsymbol{w} \otimes \boldsymbol{w}) D^{2} \rho\right)$. In [20], a similar identity for the reflected backward heat kernel $\tilde{\rho}_{(Y, s)}$ was obtained.

Lemma $3.2([20])$. There is a constant $C_{2}>0$ depending on $\Omega$ such that for $\boldsymbol{w}=\left(w_{i}\right) \in$ $\mathbb{R}^{n+1}$ with $|\boldsymbol{w}|=1$ and $\tilde{\rho}=\tilde{\rho}_{(Y, s)}(X, t)$,

$$
\frac{(\boldsymbol{w} \cdot D \tilde{\rho})^{2}}{\tilde{\rho}}+\left((I-\boldsymbol{w} \otimes \boldsymbol{w}): D^{2} \tilde{\rho}\right)+\partial_{t} \tilde{\rho} \leq C_{2}\left(\frac{|\tilde{X}-Y|}{s-t}+\frac{|\tilde{X}-Y|^{3}}{(s-t)^{2}}\right) \tilde{\rho}
$$

for $0<t<s$ and $X, Y \in N_{R / 2} \times \mathbb{R}$. 
To prove Lemma 3.2, we use the following lemma.

Lemma 3.3 (cf.[1, 11]). Let

$$
Q(X):=D \zeta(X)-(I-\boldsymbol{\nu} \otimes \boldsymbol{\nu})
$$

where $\boldsymbol{\nu}$ is the unit normal vector at $\zeta(X) \in \partial \Omega$. Then

(1) $Q(X)$ is symmetric;

(2) $Q(X) \boldsymbol{\nu}=\mathbf{0}$ for all $X \in N_{R / 2} \times \mathbb{R}$;

(3) $Q(X) \boldsymbol{e}_{n+1}=\mathbf{0}$ for all $X \in N_{R / 2} \times \mathbb{R}$, where $\boldsymbol{e}_{n+1}=(0, \ldots, 0,1)$;

(4) $|Q(X)| \leq 2|X-\zeta(X)|$ for all $X \in N_{R / 2} \times \mathbb{R}$;

(5) If $\partial \Omega \in C^{3}$, then $|D Q|$ is bounded.

For $X, Y \in N_{R / 2} \times \mathbb{R}$, by convexity

$$
|X-\zeta(X)|=\frac{1}{2}|X-\tilde{X}| \leq \frac{1}{2}(|X-Y|+|Y-\tilde{X}|) \leq|\tilde{X}-Y|
$$

thus $|Q(X)| \leq 2|\tilde{X}-Y|$.

Proof of Lemma 3.2. Since $D \zeta(X)=I-\boldsymbol{\nu} \otimes \boldsymbol{\nu}+Q(X)$ and $\tilde{X}=2 \zeta(X)-X$, we have

$$
\begin{aligned}
D|\tilde{X}-Y|^{2} & =2(I-2 \boldsymbol{\nu} \otimes \boldsymbol{\nu}+2 Q(X))(\tilde{X}-Y), \\
|D| \tilde{X}-\left.\left.Y\right|^{2}\right|^{2}= & 4|(I-2 \boldsymbol{\nu} \otimes \boldsymbol{\nu}+2 Q(X))(\tilde{X}-Y)|^{2} \\
\leq & 4|\tilde{X}-Y|^{2}+C_{3}|\tilde{X}-Y|^{3}, \\
D_{i j}|\tilde{X}-Y|^{2}= & 2 \delta_{i j}-4 \sum_{k=1}^{n}\left(\partial_{X_{j}}\left(\nu_{i} \nu_{k}\right)-\partial_{X_{j}} q_{i k}\right)\left(\tilde{X}_{k}-Y_{k}\right) \\
& +8 q_{i j}+8 \sum_{k=1}^{n}\left(q_{i k} q_{j k}-\nu_{i} \nu_{k} q_{j k}-\nu_{j} \nu_{k} q_{i k}\right),
\end{aligned}
$$

where $Q(X)=\left(q_{i j}\right)$ and $C_{3}>0$ is some constant depending on $\Omega$. By direct calculation, we have

$$
\begin{aligned}
\partial_{t} \tilde{\rho} & =\left(\frac{n}{2(s-t)}-\frac{|\tilde{X}-Y|^{2}}{4(s-t)^{2}}\right) \tilde{\rho}, \quad D \tilde{\rho}=-\frac{D|\tilde{X}-Y|^{2}}{4(s-t)} \tilde{\rho}, \\
D^{2} \tilde{\rho} & =\left(\frac{D|\tilde{X}-Y|^{2} \otimes D|\tilde{X}-Y|^{2}}{16(s-t)^{2}}-\frac{\operatorname{tr}\left(D^{2}|\tilde{X}-Y|^{2}\right)}{4(s-t)}\right) \tilde{\rho} .
\end{aligned}
$$

Using (3.6) and (3.7), we obtain (3.5).

We next prove a weighted boundary monotonicity inequality. 
Lemma 3.4. Let $\phi \in C^{1}\left([0, \infty): C^{2}(\Omega)\right)$ be a non-negative function. Then there exist positive numbers $C_{4}, C_{5}$ and $C_{6}>0$ depending on $n, \Omega$ such that

$$
\begin{aligned}
\frac{d}{d t} \int_{\Gamma_{t}} \phi\left(\rho_{1}+\rho_{2}\right) d \mathscr{H}^{n} \leq & \int_{\Gamma_{t}}\left(\rho_{1}+\rho_{2}\right)\left(\partial_{t} \phi-\Delta_{\Gamma_{t}} \phi-\left(d \phi \cdot \frac{d u}{v}\right) \frac{\partial_{t} u}{v}\right) d \mathscr{H}^{n} \\
& +\frac{1}{4} \int_{\Gamma_{t}} \phi\left(\rho_{1}+\rho_{2}\right)(\boldsymbol{f} \cdot \boldsymbol{n})^{2} d \mathscr{H}^{n} \\
& +C_{4} \mathscr{H}^{n}\left(\Gamma_{t}\right)+C_{5}(s-t)^{-\frac{3}{4}} \int_{\Gamma_{t}} \phi \rho_{2} d \mathscr{H}^{n} \\
& +C_{6} \int_{\Gamma_{t} \cap \operatorname{spt} \rho_{2}} \phi d \mathscr{H}^{n}+\int_{\partial \Gamma_{t}}\left(\rho_{1}+\rho_{2}\right)\left(D_{\Gamma_{t}} \phi \cdot \boldsymbol{\nu}\right) d \mathscr{H}^{n-1} .
\end{aligned}
$$

Proof. For $i=1,2$

$$
\begin{aligned}
\frac{d}{d t} \int_{\Gamma_{t}} \phi \rho_{i} d \mathscr{H}^{n}= & \frac{d}{d t} \int_{\Omega} \phi(x, t) \rho_{i}(x, u(x, t), t) v(x, t) d x \\
= & \int_{\Omega}\left(\partial_{t} \phi(x, t) \rho_{i}(x, u(x, t), t)\right. \\
& \left.+\phi(x, t) \partial_{x_{n+1}} \rho_{i}(x, u(x, t), t) \partial_{t} u(x, t)+\partial_{t} \rho_{i}(x, u(x, t), t)\right) v(x, t) d x \\
& +\int_{\Omega} \phi(x, t) \rho_{i}(x, u(x, t), t) \partial_{t} v(x, t) d x \\
= & \int_{\Gamma_{t}}\left(\partial_{t} \phi \rho_{i}+\phi \partial_{t} \rho_{i}+\phi \partial_{x_{n+1}} \rho_{i} \partial_{t} u\right) d \mathscr{H}^{n} \\
& +\int_{\Omega} \phi(x, t) \rho_{i}(x, u(x, t), t) \frac{d u(x, t) \cdot d\left(\partial_{t} u(x, t)\right)}{v(x, t)} d x .
\end{aligned}
$$

We consider the last term of the equation (3.9). Using integration by parts, we obtain

$$
\begin{aligned}
& \int_{\Omega} \phi(x, t) \rho_{i}(x, u(x, t), t) \frac{d u(x, t) \cdot d\left(\partial_{t} u(x, t)\right)}{v(x, t)} d x \\
= & -\int_{\Omega} \operatorname{div}\left(\phi(x, t) \rho_{i}(x, u(x, t), t) \frac{d u(x, t)}{v(x, t)}\right) \partial_{t} u(x, t) d x \\
= & -\int_{\Omega} d \phi(x, t) \cdot \frac{d u(x, t)}{v(x, t)} \rho_{i}(x, u(x, t), t) \frac{\partial_{t} u(x, t)}{v(x, t)} v(x, t) d x \\
& -\int_{\Omega} \phi(x, t)\left(d \rho_{i}(x, u(x, t), t)+\partial_{x_{n+1}} \rho_{i}(x, u(x, t), t) d u(x, t)\right) \cdot \frac{d u(x, t)}{v(x, t)} \frac{\partial_{t} u(x, t)}{v(x, t)} v(x, t) d x \\
& -\int_{\Omega} \phi(x, t) \rho_{i}(x, u(x, t), t) \operatorname{div}\left(\frac{d u(x, t)}{v(x, t)}\right) \frac{\partial_{t} u(x, t)}{v(x, t)} v(x, t) d x \\
= & -\int_{\Gamma_{t}}\left(d \phi \cdot \frac{d u}{v}\right) \rho_{i} \frac{\partial_{t} u}{v} d \mathscr{H}^{n}-\int_{\Gamma_{t}} \phi\left(\left(d \rho_{i}+\partial_{x_{n+1}} \rho_{i} d u\right) \cdot \frac{d u}{v}\right) \frac{\partial_{t} u}{v} d \mathscr{H}^{n} \\
& +\int_{\Gamma_{t}} \phi \rho_{i} h \frac{\partial_{t} u}{v} d \mathscr{H}^{n} .
\end{aligned}
$$


We note that

$$
\partial_{x_{n+1}} \rho_{i} v-\partial_{x_{n+1}} \rho_{i} \frac{|d u|^{2}}{v}-d \rho_{i} \cdot \frac{d u}{v}=\partial_{x_{n+1}} \rho_{i} \frac{1}{v}-d \rho_{i} \cdot \frac{d u}{v}=(D \rho \cdot \boldsymbol{n}) .
$$

Hence, we obtain

$$
\begin{aligned}
\frac{d}{d t} \int_{\Gamma_{t}} \phi \rho_{i} d \mathscr{H}^{n}= & \int_{\Gamma_{t}} \partial_{t} \phi \rho_{i} d \mathscr{H}^{n}+\int_{\Gamma_{t}} \phi \partial_{t} \rho_{i} d \mathscr{H}^{n}+\int_{\Gamma_{t}} \phi\left(D \rho_{i} \cdot \boldsymbol{n}\right) \frac{\partial_{t} u}{v} d \mathscr{H}^{n} \\
& -\int_{\Gamma_{t}}\left(d \phi \cdot \frac{d u}{v}\right) \rho_{i} \frac{\partial_{t} u}{v} d \mathscr{H}^{n}+\int_{\Gamma_{t}} \phi \rho_{i} h \frac{\partial_{t} u}{v} d \mathscr{H}^{n} .
\end{aligned}
$$

Using (1.1) or (2.1),

$$
\begin{aligned}
\left(\phi\left(D \rho_{i} \cdot \boldsymbol{n}\right)+\phi \rho_{i} h\right) \frac{\partial_{t} u}{v}= & \left(\phi\left(D \rho_{i} \cdot \boldsymbol{n}\right)+\phi \rho_{i} h\right)(-h+\boldsymbol{f} \cdot \boldsymbol{n}) \\
= & \phi\left(D \rho_{i} \cdot \boldsymbol{H}\right)-\phi \rho_{i}|\boldsymbol{H}|^{2}+\phi \rho_{i}\left(\left(\frac{D^{\perp} \rho_{i}}{\rho_{i}}-\boldsymbol{H}\right) \cdot \boldsymbol{n}\right)(\boldsymbol{f} \cdot \boldsymbol{n}) \\
= & -\phi \rho_{i}\left|\boldsymbol{H}-\frac{D^{\perp} \rho_{i}}{\rho_{i}}\right|^{2}+\phi \frac{\left|D^{\perp} \rho_{i}\right|^{2}}{\rho_{i}}-\phi\left(D^{\perp} \rho_{i} \cdot \boldsymbol{H}\right) \\
& +\phi \rho_{i}\left(\left(\frac{D^{\perp} \rho_{i}}{\rho_{i}}-\boldsymbol{H}\right) \cdot \boldsymbol{n}\right)(\boldsymbol{f} \cdot \boldsymbol{n}) \\
\leq & \phi \frac{\left|D^{\perp} \rho_{i}\right|^{2}}{\rho_{i}}-\phi\left(D^{\perp} \rho_{i} \cdot \boldsymbol{H}\right)+\frac{1}{4} \phi \rho_{i}(\boldsymbol{f} \cdot \boldsymbol{n})^{2}
\end{aligned}
$$

where $\boldsymbol{H}=-h \boldsymbol{n}$ and $D^{\perp} \rho_{i}=\left(D \rho_{i} \cdot \boldsymbol{n}\right) \boldsymbol{n}$ are used. Therefore,

$$
\begin{aligned}
\frac{d}{d t} \int_{\Gamma_{t}} \phi \rho_{i} d \mathscr{H}^{n} \leq & \int_{\Gamma_{t}} \partial_{t} \phi \rho_{i} d \mathscr{H}^{n}-\int_{\Gamma_{t}}\left(d \phi \cdot \frac{d u}{v}\right) \rho_{i} \frac{\partial_{t} u}{v} d \mathscr{H}^{n} \\
& +\int_{\Gamma_{t}} \phi\left(\partial_{t} \rho_{i}+\frac{\left|D^{\perp} \rho_{i}\right|^{2}}{\rho_{i}}-\left(D^{\perp} \rho_{i} \cdot \boldsymbol{H}\right)\right) d \mathscr{H}^{n} \\
& +\frac{1}{4} \int_{\Gamma_{t}} \phi \rho_{i}(\boldsymbol{f} \cdot \boldsymbol{n})^{2} d \mathscr{H}^{n} .
\end{aligned}
$$

According to the divergence theorem on $\Gamma_{t}$,

$$
\begin{aligned}
-\int_{\Gamma_{t}} \phi\left(D^{\perp} \rho_{i} \cdot \boldsymbol{H}\right) d \mathscr{H}^{n}= & -\int_{\Gamma_{t}} \phi\left(D \rho_{i} \cdot \boldsymbol{H}\right) d \mathscr{H}^{n} \\
= & \int_{\Gamma_{t}} \operatorname{div}_{\Gamma_{t}}\left(\phi D \rho_{i}\right) d \mathscr{H}^{n}-\int_{\partial \Gamma_{t}} \phi\left(D \rho_{i} \cdot \boldsymbol{\nu}\right) d \mathscr{H}^{n-1} \\
= & \int_{\Gamma_{t}} D_{\Gamma_{t}} \phi \cdot D \rho_{i} d \mathscr{H}^{n}+\int_{\Gamma_{t}} \phi\left((I-\boldsymbol{n} \otimes \boldsymbol{n}): D^{2} \rho_{i}\right) d \mathscr{H}^{n} \\
& -\int_{\partial \Gamma_{t}} \phi\left(D \rho_{i} \cdot \boldsymbol{\nu}\right) d \mathscr{H}^{n-1} \\
= & -\int_{\Gamma_{t}} \rho_{i} \Delta_{\Gamma_{t}} \phi d \mathscr{H}^{n}+\int_{\Gamma_{t}} \phi\left((I-\boldsymbol{n} \otimes \boldsymbol{n}): D^{2} \rho_{i}\right) d \mathscr{H}^{n} \\
& +\int_{\partial \Gamma_{t}}\left(\rho_{i}\left(D_{\Gamma_{t}} \phi \cdot \boldsymbol{\nu}\right)-\phi\left(D \rho_{i} \cdot \boldsymbol{\nu}\right)\right) d \mathscr{H}^{n-1} .
\end{aligned}
$$


Using (3.4) and (3.5), we obtain

$$
\frac{\left|D^{\perp} \rho_{1}\right|^{2}}{\rho_{1}}+\left((I-\boldsymbol{n} \otimes \boldsymbol{n}): D^{2} \rho_{1}\right)+\partial_{t} \rho_{1} \leq C_{7}
$$

and

$$
\frac{\left|D^{\perp} \rho_{2}\right|^{2}}{\rho_{2}}+\left((I-\boldsymbol{n} \otimes \boldsymbol{n}): D^{2} \rho_{2}\right)+\partial_{t} \rho_{2} \leq C_{8}\left(\frac{|\tilde{X}-Y|}{s-t}+\frac{|\tilde{X}-Y|^{3}}{(s-t)^{2}}\right) \rho_{2}+C_{9}
$$

for some constants $C_{7}, C_{8}, C_{9}>0$ depending on $\Omega$.

To compute the integration of (3.11), we decompose the integration as

$$
\begin{aligned}
& \int_{\Gamma_{t}} \phi \frac{C_{8}|\tilde{X}-Y|}{s-t} \rho_{2} d \mathscr{H}^{n} \\
\leq & \int_{\Gamma_{t} \cap\left\{|\tilde{X}-Y| \leq(s-t)^{\frac{1}{4}}\right\}} \phi \frac{C_{8}|\tilde{X}-Y|}{s-t} \rho_{2} d \mathscr{H}^{n} \\
& +\int_{\Gamma_{t} \cap\left\{|\tilde{X}-Y| \geq(s-t)^{\frac{1}{4}}\right\}} \phi \frac{C_{8}|\tilde{X}-Y|}{s-t} \rho_{2} d \mathscr{H}^{n} \\
= & : I_{1}+I_{2},
\end{aligned}
$$

and

$$
\begin{aligned}
& \int_{\Gamma_{t}} \phi \frac{C_{8}|\tilde{X}-Y|^{3}}{(s-t)^{2}} \rho_{2} d \mathscr{H}^{n} \\
\leq & \int_{\Gamma_{t} \cap\left\{|\tilde{X}-Y| \leq(s-t)^{\frac{5}{12}}\right\}} \phi \frac{C_{8}|\tilde{X}-Y|^{3}}{(s-t)^{2}} \rho_{2} d \mathscr{H}^{n} \\
& +\int_{\Gamma_{t} \cap\left\{|\tilde{X}-Y| \geq(s-t)^{\frac{5}{12}}\right\}} \phi \frac{C_{8}|\tilde{X}-Y|^{3}}{(s-t)^{2}} \rho_{2} d \mathscr{H}^{n} \\
= & : I_{3}+I_{4} .
\end{aligned}
$$

$I_{1}$ is estimated by

$$
I_{1} \leq C_{8}(s-t)^{-\frac{3}{4}} \int_{\Gamma_{t} \cap\left\{|\tilde{X}-Y| \leq(s-t)^{\frac{1}{4}}\right\}} \phi \rho_{2} d \mathscr{H}^{n} \leq C_{8}(s-t)^{-\frac{3}{4}} \int_{\Gamma_{t}} \phi \rho_{2} d \mathscr{H}^{n}
$$

$I_{2}$ is estimated by

$$
I_{2} \leq \frac{C_{8}}{(s-t)^{1+\frac{n}{2}}} e^{-\frac{1}{4 \sqrt{s-t}}} \int_{\Gamma_{t} \cap \operatorname{spt} \rho_{2}} \phi|\tilde{X}-Y| d \mathscr{H}^{n} \leq C_{10} R \int_{\Gamma_{t} \cap \operatorname{spt} \rho_{2}} \phi d \mathscr{H}^{n}
$$

for some constant $C_{10}>0$ depending on $n$ and $\Omega$. $I_{3}$ and $I_{4}$ are estimated as a similar manner. 
Using (3.12), (3.13), $\left.D\left(\rho_{1}+\rho_{2}\right) \cdot \boldsymbol{\nu}\right|_{\partial \Omega} \equiv 0$, and $|\tilde{X}-Y| \leq R$ when $X \in$ spt $\rho_{2}$, we compute

$$
\begin{aligned}
& \frac{d}{d t} \int_{\Gamma_{t}} \phi\left(\rho_{1}+\rho_{2}\right) d \mathscr{H}^{n} \\
\leq & \int_{\Gamma_{t}}\left(\rho_{1}+\rho_{2}\right)\left(\partial_{t} \phi-\Delta_{\Gamma_{t}} \phi-\left(d \phi \cdot \frac{d u}{v}\right) \frac{\partial_{t} u}{v}\right) d \mathscr{H}^{n} \\
& +\frac{1}{4} \int_{\Gamma_{t}}\left(\rho_{1}+\rho_{2}\right) \phi(\boldsymbol{f} \cdot \boldsymbol{n})^{2} d \mathscr{H}^{n} \\
& +\left(C_{7}+C_{9}\right) \mathscr{H}^{n}\left(\Gamma_{t}\right)+2 C_{8}(s-t)^{-\frac{3}{4}} \int_{\Gamma_{t}} \phi \rho_{2} d \mathscr{H}^{n} \\
& +C_{10}\left(R+R^{3}\right) \int_{\Gamma_{t} \cap \operatorname{spt} \rho_{2}} \phi d \mathscr{H}^{n}+\int_{\partial \Gamma_{t}}\left(\rho_{1}+\rho_{2}\right)\left(D_{\Gamma_{t}} \phi \cdot \boldsymbol{\nu}\right) d \mathscr{H}^{n-1} .
\end{aligned}
$$

For $C_{4}=C_{7}+C_{9}, C_{5}=2 C_{8}$, and $C_{6}=C_{10}\left(R+R^{3}\right)$, we obtain (3.8).

We use the following lemma to handle the boundary integral.

Lemma 3.5. Let $u$ be a classical solution of (1.1) and $v:=\sqrt{1+|d u|^{2}}$. If $\Omega$ is convex, then

$$
\left.\left(D_{\Gamma_{t}} v \cdot \boldsymbol{\nu}\right)\right|_{\partial(\Omega \times \mathbb{R})} \leq 0
$$

for all $t>0$.

Proof. Because

$$
\begin{aligned}
D_{\Gamma_{t}} v & =D v-(D v \cdot \boldsymbol{n}) \boldsymbol{n} \\
& =(d v, 0)+\frac{1}{v^{2}}(d v \cdot d u)(-d u, 1),
\end{aligned}
$$

and boundary condition of $u$,

$$
\begin{aligned}
\left.\left(D_{\Gamma_{t}} v \cdot \boldsymbol{\nu}\right)\right|_{\partial(\Omega \times \mathbb{R})} & =\left.\left((d v \cdot \nu)+\frac{1}{v^{2}}(d v \cdot d u)(-d u \cdot \nu)\right)\right|_{\partial \Omega} \\
& =\left.\frac{1}{2 v} d|d u|^{2} \cdot \nu\right|_{\partial \Omega} \\
& =\left.\frac{1}{v} B(d u, d u)\right|_{\partial \Omega},
\end{aligned}
$$

where $B$ is the second fundamental form of $\partial \Omega$. Because of the convexity of $\Omega, B(d u, d u) \leq$ 0 .

Using (3.1), (3.8), and (3.14), monotonicity of the metric is obtained as follows: 
Proposition 3.6. Let $u$ be a classical solution of (1.1) and $v:=\sqrt{1+|d u|^{2}}$. Then for $Y \in N_{R / 4} \times \mathbb{R}$ and $0<t<s$,

$$
\begin{aligned}
& \frac{d}{d t} \int_{\Gamma_{t}} v\left(\rho_{1}+\rho_{2}\right) d \mathscr{H}^{n} \\
\leq & -\int_{\Gamma_{t}}\left(\rho_{1}+\rho_{2}\right)\left(\left|A_{t}\right|^{2} v+\frac{2\left|D_{\Gamma_{t}} v\right|^{2}}{v}-d u \cdot d(\boldsymbol{f} \cdot \boldsymbol{n})\right) d \mathscr{H}^{n} \\
& +\frac{1}{4} \int_{\Gamma_{t}} v\left(\rho_{1}+\rho_{2}\right)(\boldsymbol{f} \cdot \boldsymbol{n})^{2} d \mathscr{H}^{n} \\
& +C_{4} \mathscr{H}^{n}\left(\Gamma_{t}\right)+C_{5}(s-t)^{-\frac{3}{4}} \int_{\Gamma_{t}} v\left(\rho_{1}+\rho_{2}\right) d \mathscr{H}^{n} \\
& +C_{6} \int_{\Gamma_{t} \cap \operatorname{spt} \rho_{2}} v d \mathscr{H}^{n}
\end{aligned}
$$

where $C_{4}, C_{5}, C_{6}$ are constants as in Lemma 3.4.

\section{Gradient estimates}

We deduce the integral estimates for the transport terms.

Lemma 4.1. Let $\boldsymbol{f}$ be in $L_{t}^{q} L_{x}^{p}\left(0, T_{0}, \Gamma_{t}\right)$ with $1-\frac{n}{p}-\frac{2}{q}>0$. Let $u$ be a classical solution of (1.1) and $v:=\sqrt{1+|d u|^{2}}$. Let $\eta \in L^{\infty}\left(0, T_{0}\right)$ be a nonnegative function. Then there is a constant $C_{11}>0$ depending only on $n, p, q$ and $T_{0}$ such that

$$
\begin{aligned}
& \int_{0}^{\tau} \eta d t \int_{\Gamma_{t}}\left(\rho_{1}+\rho_{2}\right) d u \cdot d(\boldsymbol{f} \cdot \boldsymbol{n}) d \mathscr{H}^{n} \\
+ & \frac{1}{4} \int_{0}^{\tau} \eta d t \int_{\Gamma_{t}} v\left(\rho_{1}+\rho_{2}\right)(\boldsymbol{f} \cdot \boldsymbol{n})^{2} d \mathscr{H}^{n} \\
\leq & \frac{1}{2} \int_{0}^{\tau} \eta d t \int_{\Gamma_{t}}\left(\rho_{1}+\rho_{2}\right)\left|A_{t}\right|^{2} v d \mathscr{H}^{n}+\int_{0}^{\tau} \eta d t \int_{\Gamma_{t}}\left(\rho_{1}+\rho_{2}\right) \frac{\left|D_{\Gamma_{t}} v\right|^{2}}{v} d \mathscr{H}^{n} \\
+ & C_{11}\|\eta\|_{L^{\infty}\left(0, T_{0}\right)}\|v\|_{L^{\infty}(\Omega \times(0, \tau))}^{3}\|\boldsymbol{f}\|_{L_{t}^{q} L_{x}^{p}\left(0, \tau, \Gamma_{t}\right)}\left(1+\|\boldsymbol{f}\|_{L_{t}^{q} L_{x}^{p}\left(0, \tau, \Gamma_{t}\right)}\right)
\end{aligned}
$$

for $0<\tau<s$, where

$$
\|\boldsymbol{f}\|_{L_{t}^{q} L_{x}^{p}\left(0, \tau, \Gamma_{t}\right)}:=\left(\int_{0}^{\tau}\left(\int_{\Gamma_{t}}|\boldsymbol{f}(X, t)|^{p} d \mathscr{H}^{n}\right)^{\frac{q}{p}} d t\right)^{\frac{1}{q}} .
$$

Proof. For simplicity, set $\bar{\rho}:=\rho_{1}+\rho_{2}$. Then

$$
\begin{aligned}
& \int_{\Gamma_{t}}\left(\rho_{1}+\rho_{2}\right)(d u \cdot d(\boldsymbol{f} \cdot \boldsymbol{n})) d \mathscr{H}^{n} \\
= & \int_{\Omega} \bar{\rho}(d u \cdot d(\boldsymbol{f} \cdot \boldsymbol{n})) v d x \\
= & -\int_{\Omega}(\bar{\rho} \Delta u v+(d u \cdot d(\bar{\rho}(x, u, t))) v+\bar{\rho}(d u \cdot d v))(\boldsymbol{f} \cdot \boldsymbol{n}) d x \\
= & -\int_{\Gamma_{t}}\left(\bar{\rho} \Delta u+(d u \cdot d(\bar{\rho}(x, u, t)))+\bar{\rho}\left(\frac{d u}{v} \cdot d v\right)\right)(\boldsymbol{f} \cdot \boldsymbol{n}) d \mathscr{H}^{n} .
\end{aligned}
$$


Here

$$
h=-\operatorname{div}\left(\frac{d u}{v}\right)=-\frac{1}{v} \Delta u+\frac{1}{v^{2}}(d u \cdot d v) ;
$$

hence,

$$
\begin{aligned}
\int_{\Gamma_{t}} \bar{\rho} d u \cdot d(\boldsymbol{f} \cdot \boldsymbol{n}) d \mathscr{H}^{n}= & \int_{\Gamma_{t}} \bar{\rho} v h(\boldsymbol{f} \cdot \boldsymbol{n}) d \mathscr{H}^{n} \\
& -2 \int_{\Gamma_{t}} \bar{\rho}\left(\frac{d u}{v} \cdot d v\right)(\boldsymbol{f} \cdot \boldsymbol{n}) d \mathscr{H}^{n} \\
& -\int_{\Gamma_{t}}(d u \cdot d(\bar{\rho}(x, u, t)))(\boldsymbol{f} \cdot \boldsymbol{n}) d \mathscr{H}^{n} \\
= & : I_{1}+I_{2}+I_{3} .
\end{aligned}
$$

$I_{1}$ is estimated by

$$
\begin{aligned}
\left|I_{1}\right| & \leq \frac{1}{2 n} \int_{\Gamma_{t}} \bar{\rho} h^{2} v d \mathscr{H}^{n}+\frac{n}{2} \int_{\Gamma_{t}} \bar{\rho} v(\boldsymbol{f} \cdot \boldsymbol{n})^{2} d \mathscr{H}^{n} \\
& \leq \frac{1}{2} \int_{\Gamma_{t}} \bar{\rho}\left|A_{t}\right|^{2} v d \mathscr{H}^{n}+\frac{n}{2} \int_{\Gamma_{t}} \bar{\rho} v(\boldsymbol{f} \cdot \boldsymbol{n})^{2} d \mathscr{H}^{n}
\end{aligned}
$$

because $h^{2} \leq n\left|A_{t}\right|^{2}$.

Note that $D_{\Gamma_{t}} v=D v-(D v \cdot \boldsymbol{n}) \boldsymbol{n}$

$$
\begin{aligned}
\left|D_{\Gamma_{t}} v\right|^{2} & =|D v|^{2}-(D v \cdot \boldsymbol{n})^{2} \\
& =|d v|^{2}-\frac{1}{v^{2}}(d u \cdot d v)^{2} \quad(\because D v=(d v, 0)) \\
& \geq|d v|^{2}-\frac{1}{v^{2}}|d u|^{2}|d v|^{2} \\
& =\frac{1}{v^{2}}|d v|^{2} .
\end{aligned}
$$

Therefore,

$$
\begin{aligned}
\left|I_{2}\right| & \leq \int_{\Gamma_{t}} \bar{\rho} \frac{|d u|^{2}|d v|^{2}}{v^{5}} d \mathscr{H}^{n}+\int_{\Gamma_{t}} \bar{\rho} v^{3}(\boldsymbol{f} \cdot \boldsymbol{n})^{2} d \mathscr{H}^{n} \\
& \leq \int_{\Gamma_{t}} \overline{\bar{\rho}} \frac{\left|D_{\Gamma_{t}} v\right|^{2}}{v} d \mathscr{H}^{n}+\int_{\Gamma_{t}} \bar{\rho} v^{3}(\boldsymbol{f} \cdot \boldsymbol{n})^{2} d \mathscr{H}^{n} .
\end{aligned}
$$

In the following, we derive the integral estimates for the transport terms. Using the Hölder inequality,

$$
\begin{aligned}
& \left|\int_{0}^{\tau} \eta d t \int_{\Gamma_{t}} \bar{\rho}(\boldsymbol{f} \cdot \boldsymbol{n})^{2} v^{3} d \mathscr{H}^{n}\right| \\
\leq & \|\eta\|_{L^{\infty}\left(0, T_{0}\right)}\|v\|_{L^{\infty}(\Omega \times(0, \tau))}^{3}\left(\int_{0}^{s} d t\left(\int_{\Gamma_{t}} \bar{\rho}^{p^{\prime}} d \mathscr{H}^{n}\right)^{\frac{q^{\prime}}{p^{\prime}}}\right)^{\frac{1}{q^{\prime}}}\|\boldsymbol{f}\|_{L_{t}^{q} L_{x}^{p}\left(0, \tau, \Gamma_{t}\right)}^{2},
\end{aligned}
$$


where $\frac{2}{p}+\frac{1}{p^{\prime}}=1$ and $\frac{2}{q}+\frac{1}{q^{\prime}}=1$. Using the convexity of $\Omega,|\tilde{X}-Y| \geq|X-Y|$; hence,

$$
\begin{aligned}
\int_{\Gamma_{t}} \bar{\rho}^{p^{\prime}} d \mathscr{H}^{n} & \leq \frac{2^{p^{\prime}-1}}{(4 \pi(s-t))^{\frac{n p^{\prime}}{2}}} \int_{\Gamma_{t}} \exp \left(-\frac{p^{\prime}|X-Y|^{2}}{4(s-t)}\right) d \mathscr{H}^{n} \\
& \leq C_{12}(s-t)^{-\frac{n p^{\prime}}{2}+\frac{n}{2}}
\end{aligned}
$$

where $C_{12}>0$ is some constant depending only on $n$ and $p$. Therefore,

$$
\left(\int_{0}^{s} d t\left(\int_{\Gamma_{t}} \bar{\rho}^{p^{\prime}} d \mathscr{H}^{n}\right)^{\frac{q^{\prime}}{p^{\prime}}}\right)^{\frac{1}{q^{\prime}}}<\infty
$$

if $-\frac{n q^{\prime}}{2}+\frac{n q^{\prime}}{2 p^{\prime}}>-1$, which provides $1-\frac{n}{p}-\frac{2}{q}>0$. Using (4.2) and (4.3), we obtain

$$
\begin{aligned}
\int_{0}^{\tau} \eta\left(\left|I_{1}\right|+\left|I_{2}\right|\right) d t \leq & \frac{1}{2} \int_{0}^{\tau} \eta d t \int_{\Gamma_{t}} \bar{\rho}\left|A_{t}\right|^{2} v d \mathscr{H}^{n} \\
& +\int_{0}^{\tau} \eta d t \int_{\Gamma_{t}} \bar{\rho} \frac{\left|D_{\Gamma_{t}} v\right|^{2}}{v} d \mathscr{H}^{n} \\
& +C_{13}\|\eta\|_{L^{\infty}\left(0, T_{0}\right)}\|v\|_{L^{\infty}(\Omega \times(0, \tau))}^{3}\|\boldsymbol{f}\|_{L_{t}^{q} L_{x}^{p}\left(0, \tau, \Gamma_{t}\right)}^{2}
\end{aligned}
$$

for a positive constant $C_{13}>0$ depending only on $n, p, q$ and $T_{0}$.

Because

$$
|d u \cdot d(\bar{\rho}(x, u, t))|=\left.|d u \cdot d \bar{\rho}+| d u\right|^{2} \bar{\rho}_{x_{n+1}}\left|\leq v^{2}\right| D \bar{\rho} \mid,
$$

we obtain

$$
\left|I_{3}\right| \leq \int_{\Gamma_{t}} v^{2}|D \bar{\rho}||\boldsymbol{f} \cdot \boldsymbol{n}| d \mathscr{H}^{n} .
$$

Then using the Hölder inequality,

$$
\begin{aligned}
\int_{0}^{\tau} \eta\left|I_{3}\right| d t \leq & \|\eta\|_{L^{\infty}\left(0, T_{0}\right)}\|v\|_{L^{\infty}(\Omega \times(0, \tau))}^{2} \\
& \times\left(\int_{0}^{s} d t\left(\int_{\Gamma_{t}}|D \bar{\rho}|^{p^{\prime}} d \mathscr{H}^{n}\right)^{\frac{q^{\prime}}{p^{\prime}}}\right)^{\frac{1}{q^{\prime}}}\|\boldsymbol{f}\|_{L_{t}^{q} L_{x}^{p}\left(0, \tau, \Gamma_{t}\right)},
\end{aligned}
$$

where $\frac{1}{p}+\frac{1}{p^{\prime}}=1$ and $\frac{1}{q}+\frac{1}{q^{\prime}}=1$. Using the convexity of $\Omega$,

$$
|D \bar{\rho}| \leq C_{14} \frac{1}{(s-t)^{\frac{1}{2}+\frac{n}{2}}} \exp \left(-\frac{|X-Y|^{2}}{8(s-t)}\right),
$$

where $C_{14}>0$ is some constant depending only on $n$ and $p$. Therefore,

$$
\int_{\Gamma_{t}}|D \bar{\rho}|^{p^{\prime}} d \mathscr{H}^{n} \leq C_{14}(s-t)^{-\frac{p^{\prime}}{2}-\frac{n p^{\prime}}{2}+\frac{n}{2}}
$$

hence,

$$
\left(\int_{0}^{s} d t\left(\int_{\Gamma_{t}}|D \bar{\rho}|^{p^{\prime}} d \mathscr{H}^{n}\right)^{\frac{q^{\prime}}{p^{\prime}}}\right)^{\frac{1}{q^{\prime}}}<\infty
$$


if $-\frac{q^{\prime}}{2}-\frac{n q^{\prime}}{2}+\frac{n q^{\prime}}{2 p^{\prime}}>-1$, which provides $1-\frac{n}{p}-\frac{2}{q}>0$. Therefore, using (4.5) we obtain

$$
\int_{0}^{\tau} \eta\left|I_{3}\right| d t \leq C_{15}\|\eta\|_{L^{\infty}\left(0, T_{0}\right)}\|v\|_{L^{\infty}(\Omega \times(0, \tau))}^{2}\|\boldsymbol{f}\|_{L_{t}^{q} L_{x}^{p}\left(0, \tau, \Gamma_{t}\right)}
$$

for some constant $C_{15}>0$ depending only on $n, p, q$ and $T_{0}$. Combining (4.4) and (4.6), we obtain (4.1).

Proof of Theorem 2.2. Let $T \in\left(0, T_{0}\right)$. We denote

$$
M_{T}:=\sup _{0<t<T}\|v(\cdot, t)\|_{L^{\infty}(\Omega)} .
$$

We first consider the interior gradient estimates. By arguments similar to that in Proposition 3.6 and Lemma 4.1 with $\eta \equiv 1$, for $Y=\left(y, y_{n+1}\right) \in\left(\Omega \backslash N_{R / 6}\right) \times \mathbb{R}$ and $0<\tau<s \leq T$,

$$
\begin{aligned}
& \frac{d}{d t} \int_{\Gamma_{t}} v \rho_{1} d \mathscr{H}^{n} \\
\leq & -\int_{\Gamma_{t}} \rho_{1}\left(\left|A_{t}\right|^{2} v+\frac{2\left|D_{\Gamma_{t}} v\right|^{2}}{v}-d u \cdot d(\boldsymbol{f} \cdot \boldsymbol{n})\right) d \mathscr{H}^{n} \\
& +\frac{1}{4} \int_{\Gamma_{t}} v \rho_{1}(\boldsymbol{f} \cdot \boldsymbol{n})^{2} d \mathscr{H}^{n}+C_{4} \mathscr{H}^{n}\left(\Gamma_{t}\right)
\end{aligned}
$$

and

$$
\begin{aligned}
& \int_{0}^{\tau} d t \int_{\Gamma_{t}} \rho_{1} d u \cdot d(\boldsymbol{f} \cdot \boldsymbol{n}) d \mathscr{H}^{n}+\frac{1}{4} \int_{0}^{\tau} d t \int_{\Gamma_{t}} v \rho_{1}(\boldsymbol{f} \cdot \boldsymbol{n})^{2} d \mathscr{H}^{n} \\
& \leq \frac{1}{2} \int_{0}^{\tau} d t \int_{\Gamma_{t}} \rho_{1}\left|A_{t}\right|^{2} v d \mathscr{H}^{n}+\int_{0}^{\tau} d t \int_{\Gamma_{t}} \rho_{1} \frac{\left|D_{\Gamma_{t}} v\right|^{2}}{v} d \mathscr{H}^{n} \\
& \quad+C_{11}\|v\|_{L^{\infty}(\Omega \times(0, \tau))}^{3}\|\boldsymbol{f}\|_{L_{t}^{q} L_{x}^{p}\left(0, \tau, \Gamma_{t}\right)}\left(1+\|\boldsymbol{f}\|_{L_{t}^{q} L_{x}^{p}\left(0, \tau, \Gamma_{t}\right)}\right)
\end{aligned}
$$

where the positive constants $C_{4}$ and $C_{11}$ are same as in Lemma 3.4 and Lemma 4.1 respectively. Using (4.7) and (4.8) we have

$$
\begin{aligned}
& \int_{\Gamma_{\tau}} v(x, \tau) \rho_{1}(X, \tau) d \mathscr{H}^{n}-\int_{\Gamma_{0}} v(x, 0) \rho_{1}(X, 0) d \mathscr{H}^{n} \\
\leq & C_{4} \int_{0}^{\tau} \int_{\Omega} v d x d t+C_{11}\|v\|_{L^{\infty}(\Omega \times(0, \tau))}^{3}\|\boldsymbol{f}\|_{L_{t}^{q} L_{x}^{p}\left(0, \tau, \Gamma_{t}\right)}\left(1+\|\boldsymbol{f}\|_{L_{t}^{q} L_{x}^{p}\left(0, \tau, \Gamma_{t}\right)}\right) \\
\leq & C_{4}|\Omega| M_{T} s+C_{11} M_{T}^{3}\|\boldsymbol{f}\|_{L_{t}^{q} L_{x}^{p}\left(0, s, \Gamma_{t}\right)}\left(1+\|\boldsymbol{f}\|_{L_{t}^{q} L_{x}^{p}\left(0, s, \Gamma_{t}\right)}\right) .
\end{aligned}
$$

Passing $\tau \rightarrow s$ we obtain

$$
\begin{aligned}
v(y, s) \leq & \int_{\Omega} v_{0}^{2} \rho_{1}(X, 0) d x+C_{4}|\Omega| M_{T} s \\
& +C_{11} M_{T}^{3}\|\boldsymbol{f}\|_{L_{t}^{q} L_{x}^{p}\left(0, s, \Gamma_{t}\right)}\left(1+\|\boldsymbol{f}\|_{L_{t}^{q} L_{x}^{p}\left(0, s, \Gamma_{t}\right)}\right) \\
\leq & \left\|v_{0}\right\|_{\infty}^{2}+C_{4}|\Omega| M_{T} s+C_{11} M_{T}^{3}\|\boldsymbol{f}\|_{L_{t}^{q} L_{x}^{p}\left(0, s, \Gamma_{t}\right)}\left(1+\|\boldsymbol{f}\|_{L_{t}^{q} L_{x}^{p}\left(0, s, \Gamma_{t}\right)}\right)
\end{aligned}
$$

where $v_{0}:=\sqrt{1+\left|d u_{0}\right|^{2}}$. 
Next we consider the boundary gradient estimates. By Proposition [3.6, for $Y=$ $\left(y, y_{n+1}\right) \in N_{R / 4} \times \mathbb{R}, 0<\tau<s \leq T$, and a non-negative function $\eta=\eta(t) \in L^{\infty}\left(0, T_{0}\right)$,

$$
\begin{aligned}
& \eta \frac{d}{d t} \int_{\Gamma_{t}} v\left(\rho_{1}+\rho_{2}\right) d \mathscr{H}^{n} \\
\leq & -\eta \int_{\Gamma_{t}}\left(\rho_{1}+\rho_{2}\right)\left(\left|A_{t}\right|^{2} v+\frac{2\left|D_{\Gamma_{t}} v\right|^{2}}{v}-d u \cdot d(\boldsymbol{f} \cdot \boldsymbol{n})\right) d \mathscr{H}^{n} \\
& +\frac{\eta}{4} \int_{\Gamma_{t}} v\left(\rho_{1}+\rho_{2}\right)(\boldsymbol{f} \cdot \boldsymbol{n})^{2} d \mathscr{H}^{n} \\
& +C_{4} \eta \mathscr{H}^{n}\left(\Gamma_{t}\right)+C_{5} \eta(s-t)^{-\frac{3}{4}} \int_{\Gamma_{t}} v\left(\rho_{1}+\rho_{2}\right) d \mathscr{H}^{n} \\
& +C_{6} \eta \int_{\Gamma_{t} \cap \operatorname{spt} \rho_{2}} v d \mathscr{H}^{n} .
\end{aligned}
$$

Let

$$
\eta(\tau):=\exp \left(-C_{5} \int_{0}^{\tau}(s-t)^{-\frac{3}{4}} d t\right)=\exp \left(-C_{5}\left(s^{\frac{1}{4}}-(s-\tau)^{\frac{1}{4}}\right)\right) .
$$

Note that $\|\eta\|_{\infty}=1$. Then by Lemma 4.1, we have

$$
\begin{aligned}
& \quad \exp \left(-C_{5}\left(s^{\frac{1}{4}}-(s-\tau)^{\frac{1}{4}}\right)\right) \int_{\Gamma_{\tau}} v(x, \tau)\left(\rho_{1}+\rho_{2}\right)(X, \tau) d \mathscr{H}^{n} \\
& \quad-\int_{\Gamma_{0}} v(x, 0)\left(\rho_{1}+\rho_{2}\right)(X, 0) d \mathscr{H}^{n} \\
& \leq C_{4} \int_{0}^{\tau} \eta d t \int_{\Omega} v d x+C_{6} \int_{0}^{\tau} \eta d t \int_{\Omega} v^{2} d x \\
& \quad+C_{11}\|v\|_{L^{\infty}(\Omega \times(0, \tau))}^{3}\|\boldsymbol{f}\|_{L_{t}^{q} L_{x}^{p}\left(0, \tau, \Gamma_{t}\right)}\left(1+\|\boldsymbol{f}\|_{L_{t}^{q} L_{x}^{p}\left(0, \tau, \Gamma_{t}\right)}\right) \\
& \leq C_{4}|\Omega| M_{T} s+C_{6}|\Omega| M_{T}^{2} s+C_{11} M_{T}^{3}\|\boldsymbol{f}\|_{L_{t}^{q} L_{x}^{p}\left(0, s, \Gamma_{t}\right)}\left(1+\|\boldsymbol{f}\|_{L_{t}^{q} L_{x}^{p}\left(0, s, \Gamma_{t}\right)}\right) .
\end{aligned}
$$

Passing $\tau \rightarrow s$ we obtain

$$
\begin{aligned}
\exp \left(-C_{5} s^{\frac{1}{4}}\right) v(y, s) \leq & 2\left\|v_{0}\right\|_{\infty}^{2}+C_{4}|\Omega| M_{T} s+C_{6}|\Omega| M_{T}^{2} s \\
& +C_{11} M_{T}^{3}\|\boldsymbol{f}\|_{L_{t}^{q} L_{x}^{p}\left(0, s, \Gamma_{t}\right)}\left(1+\|\boldsymbol{f}\|_{L_{t}^{q} L_{x}^{p}\left(0, s, \Gamma_{t}\right)}\right)
\end{aligned}
$$

where $v_{0}:=\sqrt{1+\left|d u_{0}\right|^{2}}$. Compared (4.9) with (4.10), we obtain for all $y \in \bar{\Omega}$ and $0<s \leq T$

$$
\exp \left(-C_{5} s^{\frac{1}{4}}\right) v(y, s) \leq 2\left\|v_{0}\right\|_{\infty}^{2}+C_{16}(s) M_{T}^{3}
$$

where

$$
C_{16}(s):=\left(C_{4}+C_{6}\right)|\Omega| s+C_{11}\|\boldsymbol{f}\|_{L_{t}^{q} L_{x}^{p}\left(0, s, \Gamma_{t}\right)}\left(1+\|\boldsymbol{f}\|_{L_{t}^{q} L_{x}^{p}\left(0, s, \Gamma_{t}\right)}\right) .
$$

Note that $C_{16}(s)$ is monotone increasing and $C_{16}(s) \rightarrow 0$ as $s \downarrow 0$.

Now, select $(y, s)$ such that $M_{T}=v(y, s)$ and $Y=(y, u(y, s))$. Then, by monotonicity of $C_{16}(s)$

$$
C_{16}(T) M_{T}^{3}-\exp \left(-C_{5} T^{\frac{1}{4}}\right) M_{T}+2\left\|v_{0}\right\|_{\infty}^{2} \geq 0
$$


When $4\left\|v_{0}\right\|_{\infty}^{2} \leq M_{T} \leq 5\left\|v_{0}\right\|_{\infty}^{2}$ for some $T>0$. Then by (4.12) we have

$$
C_{16}(T) \geq \frac{\exp \left(-C_{5} T^{\frac{1}{4}}\right)}{M_{T}^{2}}-\frac{2\left\|v_{0}\right\|_{\infty}^{2}}{M_{T}^{3}} \geq \frac{C_{17}(T)}{\left\|v_{0}\right\|_{\infty}^{4}}
$$

where

$$
C_{17}(T)=\frac{\exp \left(-C_{5} T^{\frac{1}{4}}\right)}{25}-\frac{1}{32}
$$

Thus, we have

$$
M_{T_{1}} \leq 4\left\|v_{0}\right\|_{\infty}^{2}=1+\left\|d u_{0}\right\|_{\infty}^{2}
$$

where $T_{1}$ is sufficiently small constant satisfying $C_{17}(T)>0$ and $C_{16}(T)<\frac{C_{17}(T)}{\left\|v_{0}\right\|_{\infty}^{4}}$.

\section{Existence of ClASSiCAL SOLUTiOnS}

Finally, we prove Theorem 2.3. To use the Schauder estimates, we provide the following:

Lemma 5.1. Let $T>0$ and $u \in C^{2,1}\left(Q_{T}\right)$ be a solution of (1.1). Then

$$
\sup _{Q_{T}}|u| \leq \sup _{\Omega \times \mathbb{R} \times[0,1]}|\boldsymbol{f}| T+\sup _{\Omega}\left|u_{0}\right| \text {. }
$$

Proof. We set $w(x, t)=\sup _{\Omega \times \mathbb{R} \times[0,1]}|\boldsymbol{f}| t+\sup _{\Omega}\left|u_{0}\right|$. We note that

$$
\partial_{t} w \geq \sqrt{1+|d w|^{2}} \operatorname{div}\left(\frac{d w}{\sqrt{1+|d w|^{2}}}\right)+\boldsymbol{f}(x, w, t) \cdot(-d w, 1) .
$$

Using the comparison principle, we determine that

$$
w \geq u, \quad(x, t) \in Q_{T} .
$$

Similarly to the above argument,

$$
u \geq-w, \quad(x, t) \in Q_{T} .
$$

Hence, we obtain (5.1).

Proof of Theorem 2.3. Fix $\alpha \in(0,1)$. We assume that $u_{0} \in C^{2, \alpha}(\Omega)$ and let $T>0$, which is given by Theorem 2.2. Let $\beta \in(0, \alpha]$ and we set $X:=C^{1, \beta}\left(Q_{T}\right)$. We consider the following linear parabolic type equation:

$$
\left\{\begin{aligned}
\partial_{t} u & =\sum_{i, j=1}^{n} a_{i j}(d w) \partial_{x_{i} x_{j}} u+f(x, w, t) \cdot(-d u, 1) & & \text { in } Q_{T}, \\
\left.d u \cdot \nu\right|_{\partial \Omega} & =0, & & \\
\left.u\right|_{t=0} & =u_{0}, & & \text { on } \Omega,
\end{aligned}\right.
$$

where $w \in X$ and $a_{i j}(r)=\left(\delta_{i j}-\frac{r_{i} r_{j}}{1+|r|^{2}}\right)$ for $r=\left(r_{1}, \ldots, r_{n}\right)$. Because

$$
\begin{aligned}
\left\|a_{i j}(d w)\right\|_{C^{\alpha \beta}\left(Q_{T}\right)} & \leq\left\|a_{i j}(d w)\right\|_{C^{\beta}\left(Q_{T}\right)} \\
& \leq\left\|a_{i j}\right\|_{C^{1}\left(\mathbb{R}^{n}\right)}\|d w\|_{C^{\beta}\left(Q_{T}\right)} \leq\left\|a_{i j}\right\|_{C^{1}\left(\mathbb{R}^{n}\right)}\|w\|_{X}
\end{aligned}
$$


for any $w \in X,($ (5.2) $)$ is uniformly parabolic in $Q_{T}$. Note that $\left\|a_{i j}\right\|_{C^{1}\left(\mathbb{R}^{n}\right)}<\infty$. Using (2.4), we obtain

$$
\|\boldsymbol{f}(\cdot, w, \cdot)\|_{C^{\alpha \beta}\left(Q_{T}\right)} \leq K\|w\|_{C^{\beta}\left(Q_{T}\right)} \leq K\|w\|_{X}
$$

for any $w \in X$. Hence, for any $w \in X$ there exists a unique solution $u_{w} \in C^{2, \alpha \beta}\left(Q_{T}\right) \subset X$ of (5.2) such that

$$
\left\|u_{w}\right\|_{C^{2, \alpha \beta}\left(Q_{T}\right)} \leq C_{18},
$$

where $C_{18}>0$ depends only on $n, \alpha, \beta,\|w\|_{X},\left\|u_{0}\right\|_{C^{2, \alpha}(\Omega)}$ and $K$ (see [17, Theorem 4.5.3]).

We define $A: X \rightarrow X$ as $A w=u_{w}$. Note that $A$ is continuous and compact. We show that

$$
S:=\{u \mid u=\sigma A u \text { in } X, \text { for some } \sigma \in[0,1]\}
$$

is bounded in $X$. If $u \in S$, then

$$
\left\{\begin{aligned}
\partial_{t} u & =\sum_{i, j=1}^{n} a_{i j}(d u) \partial_{x_{i} x_{j}} u+f(x, u, t) \cdot(-d u, \sigma), & & \text { in } Q_{T}, \\
\left.d u \cdot \nu\right|_{\partial \Omega} & =0, & & \text { on } \Omega . \\
\left.u\right|_{t=0} & =\sigma u_{0}, & & \text { on }
\end{aligned}\right.
$$

According to Theorem 2.2 ,

$$
\sup _{Q_{T}}|d u| \leq 4\left(1+\left\|d u_{0}\right\|_{\infty}^{2}\right)
$$

Because $d u \cdot \nu=0$ on $\partial \Omega$, we can use similar arguments to the interior Schauder estimates (cf. [17, Theorem 6.2.1]); hence,

$$
\|d u\|_{C^{\beta}\left(Q_{T}\right)} \leq C_{19},
$$

where $C_{19}=C_{19}$ is a positive constant depending only on $n, \sup _{Q_{T}}|u|,\left\|d u_{0}\right\|_{C^{\alpha}(\Omega)}$, $\sup _{\Omega \times \mathbb{R} \times[0, T]}|\boldsymbol{f}|$, and $\partial \Omega$.

Using the same argument as (5.5),

$$
\|u\|_{X} \leq\|u\|_{C^{2, \alpha \beta}\left(Q_{T}\right)} \leq C_{20},
$$

where $C_{20}=C_{20}\left(n, \alpha,\left\|u_{0}\right\|_{C^{2, \alpha}(\Omega)}, C_{19}, K\right)>0$ (see [17]). According to (5.7), (5.8), and (5.9),$C_{20}$ depends only on $n, \alpha,\left\|u_{0}\right\|_{C^{2, \alpha}(\Omega)}, \sup _{\Omega}\left|d u_{0}\right|$ and $K$. Thus, $S$ is bounded in $X$. According to the Leray-Schauder fixed point theorem, there exists a solution $u \in C^{2, \alpha}\left(Q_{T}\right)$ of (1.1).

We return to the assumption that $u_{0}$ is a Lipschitz function with a Lipschitz constant $L>0$. Set $\varepsilon>0$. We choose smooth functions $u_{0}^{k}$ converging uniformly to $u_{0}$ on $\Omega$. We note that according to Theorem 2.2 ,

$$
\sup _{Q_{T}}\left|d u^{k}\right| \leq 4\left(1+L^{2}\right)
$$

for all $k \geq 1$. Using an argument similar to (5.8), (5.9) and the interior Schauder estimates, there exists $C_{21}=C_{21}(n, \alpha, L, \varepsilon, K)>0$ such that

$$
\sup _{k}\left\|u^{k}\right\|_{C^{2, \alpha}\left(Q_{T}^{\varepsilon}\right)} \leq C_{21} \text {. }
$$

where $u^{k}$ is the solution of (1.1) with $u^{k}(x, 0)=u_{0}^{k}(x)$ in $\Omega$. Note that $\varepsilon=\operatorname{dist}\left(Q_{T}^{\varepsilon}, \partial Q_{T}\right)$. 


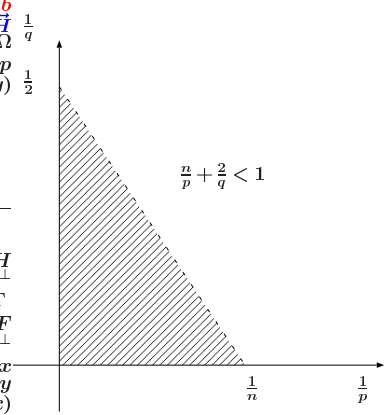

Figure 3. For $p$ and $q$ not belonging to the above oblique line, there is a transport term such that the gradient of some solution of (1.1) is not bounded.

Hence, for any $\varepsilon>0$, passing to a subsequence if necessary, $\left\{u^{k}\right\}_{k=1}^{\infty}$ converges to a classical solution $u$ in $Q_{T}^{\varepsilon}$ and we obtain (2.5). Therefore, by diagonal arguments, we obtain the solution $u \in C\left(\overline{Q_{T}}\right) \cap C^{2,1}\left(Q_{T}^{\varepsilon}\right)$ of (1.1). The comparison principle implies the uniqueness of the solution of (1.1). Thus, we have proved Theorem 2.3.

\section{Optimality}

We now discuss optimality about the assumption to transport terms for the gradient estimates. We present some transport term $\boldsymbol{f}$ such that the gradient of some solution of (1.1) is not bounded and $\|\boldsymbol{f}\|_{L_{t}^{q} L_{x}^{p}\left(0,1, \Gamma_{t}\right)}<\infty$ for some $p, q$ satisfying $\frac{n}{p}+\frac{2}{q}>1$ (see Figure 3).

Let $\phi=\phi(\xi)$ be a smooth function on $\mathbb{R}^{n}$ compactly supported on $\Omega$. We seek the transport term such that $u(x, t)=(1-t)^{\alpha} \phi(x / \sqrt{1-t})$ is a solution of (1.1) for $\alpha \in \mathbb{R}$. By direct calculation, we obtain

$$
\begin{aligned}
d u(x, t) & =(1-t)^{\alpha-\frac{1}{2}} d_{\xi} \phi, \quad d^{2} u(x, t)=(1-t)^{\alpha-1} d_{\xi}^{2} \phi \\
\partial_{t} u(x, t) & =(1-t)^{\alpha-1}\left(-\alpha \phi+\frac{1}{2} d_{\xi} \phi \cdot \frac{x}{\sqrt{1-t}}\right) .
\end{aligned}
$$

Note that if $\alpha<\frac{1}{2}$, then $d u$ is not bounded. If $u(x, t)=(1-t)^{\alpha} \phi(x / \sqrt{1-t})$ is a solution of (1.1), then we obtain

$$
\begin{aligned}
\boldsymbol{f}(x, u, t) \cdot(-d u, 1)= & (1-t)^{\alpha-1}\left(-\alpha \phi+\frac{1}{2} d_{\xi} \phi \cdot \frac{x}{\sqrt{1-t}}\right) \\
& -(1-t)^{\alpha-1} \Delta_{\xi} \phi+(1-t)^{3 \alpha-2} \frac{d_{\xi}^{2} \phi:\left(d_{\xi} \phi \otimes d_{\xi} \phi\right)}{1+(1-t)^{2 \alpha-1}\left|d_{\xi} \phi\right|^{2}} .
\end{aligned}
$$

Thus, for $p, q \geq 1$ and $\alpha<\frac{1}{2}$ if $t$ is sufficiently near to 1 , we obtain

$$
C_{22}(\phi)(1-t)^{3 \alpha-2+\frac{n}{2 p}+\frac{2 \alpha-1}{p}} \leq\left(\int_{\Gamma_{t}}|\boldsymbol{f}|^{p} d \mathscr{H}^{n}\right)^{\frac{1}{p}} \leq C_{23}(\phi)(1-t)^{3 \alpha-2+\frac{n}{2 p}+\frac{2 \alpha-1}{p}}
$$

for some constants $C_{22}(\phi), C_{23}(\phi)>0$ since $\frac{1}{2(1-t)^{2 \alpha-1}|d \phi|^{2}} \leq \frac{1}{1+(1-t)^{2 \alpha-1}|d \phi|^{2}} \leq \frac{1}{(1-t)^{2 \alpha-1}|d \phi|^{2}}$. 
When $1+\varepsilon_{0}:=\frac{n}{p}+\frac{2}{q}>1$ and $\|\boldsymbol{f}\|_{L_{t}^{q} L_{x}^{p}\left(0,1, \Gamma_{t}\right)}<\infty$, then

$$
\int_{0}^{1}(1-t)^{\left(3 \alpha-2+\frac{n}{2 p}+\frac{2 \alpha-1}{p}\right) q} d t<\infty
$$

hence we obtain $\alpha>\frac{1}{2}-\frac{\varepsilon_{0}}{2}\left(3+\frac{2}{p}\right)^{-1}$. For $\alpha_{0}:=\frac{1}{2}-\frac{\varepsilon_{0}}{4}\left(3+\frac{2}{p}\right)^{-1}$, let $\boldsymbol{f}$ be given by (6.1) with $\alpha=\alpha_{0}$. Then $u(t, x):=(1-t)^{\alpha} \phi(x / \sqrt{1-t})$ is a solution of (6.1), $\|\boldsymbol{f}\|_{L_{t}^{q} L_{x}^{p}\left(0,1, \Gamma_{t}\right)}<\infty$ for some $p, q$ satisfying $\frac{n}{p}+\frac{2}{q}>1$ and $d u$ is not bounded on $\Omega \times(0,1)$.

\section{FINAL REMARK}

By our argument, we do not obtain a time global classical solution of (1.1). Indeed, when $f \not \equiv 0$, the maximum existence time in Theorem 2.3 cannot be taken infinity. On the other hand, Huisken [12, Theorem 1.1] proved that there exists a time global solution of (1.1) under $\boldsymbol{f} \equiv 0$ and $u_{0} \in C^{2, \alpha}(\bar{\Omega})$ and the solution converges to some constant function as $t \rightarrow \infty$. In the case of $\boldsymbol{f} \not \equiv 0$, a priori time global gradient bounds is not known hence we do not show the global existence for solutions of (1.1). It is expected that with the assumption (2.4) there is a time global solution of (1.1) and that solution converges to a solution of the prescribed mean curvature equation $\boldsymbol{H}=-(\boldsymbol{f} \cdot \boldsymbol{n}) \boldsymbol{n}$.

To apply our results for $\Gamma_{t}$ in (1.4), the velocity vector $f$ in (1.4) needs to be smooth enough. On the other hand, if $\boldsymbol{f}$ is a weak solution of (1.4), namely $\boldsymbol{f} \in L_{t}^{2}\left(H_{x}^{1}\right)$, then $\boldsymbol{f}$ does not satisfy our assumption. When we consider (1.4) with $\boldsymbol{f} \in L_{t}^{2}\left(H_{x}^{1}\right)$, we need to study the relationship between the velocity vector $f$ and the phase boundary $\Gamma_{t}$.

\section{ACKNOWLEDGMENTS}

The authors are grateful to the referee for his or her helpful comments. This work was supported by JSPS KAKENHI Grant Numbers 25800084, 25247008, 16K17622, 17J02386. The second author is supported by JSPS Research Fellowships for Young Scientists.

\section{REFERENCES}

[1] W. K. Allard, On the first variation of a varifold: boundary behavior, Ann. of Math. (2) 101 (1975), 418-446.

[2] S. J. Altschuler and L. F. Wu, Translating surfaces of the non-parametric mean curvature flow with prescribed contact angle, Calc. Var. Partial Differential Equations 2 (1994), 101-111.

[3] J. A. Buckland, Mean curvature flow with free boundary on smooth hypersurfaces, J. Reine Angew. Math. 586 (2005), 71-90.

[4] T. H. Colding and W. P. Minicozzi, II, Sharp estimates for mean curvature flow of graphs, J. Reine Angew. Math. 574 (2004), 187-195.

[5] K. Ecker, Regularity theory for mean curvature flow, Vol. 57, Birkhäuser Boston, Inc., Boston, MA, 2004.

[6] K. Ecker and G. Huisken, Interior curvature estimates for hypersurfaces of prescribed mean curvature, Ann. Inst. H. Poincaré Anal. Non Linéaire 6 (1989), 251-260.

[7] K. Ecker, and G. Huisken, Mean curvature evolution of entire graphs, Ann. of Math. (2), 130 (1989), 453-471.

[8] K. Ecker and G. Huisken, Interior estimates for hypersurfaces moving by mean curvature, Invent. Math. 105 (1991), 547-569.

[9] N. Edelen, Convexity estimates for mean curvature flow with free boundary, Adv. Math. 294 (2016), $1-36$.

[10] N. Edelen, The free-boundary Brakke flow, arXiv preprint arXiv:1602.03614, 2016. 
[11] M. Grüter and J. Jost, Allard type regularity results for varifolds with free boundaries, Ann. Scuola Norm. Sup. Pisa Cl. Sci. (4) 13 (1986), 129-169.

[12] G. Huisken, Nonparametric mean curvature evolution with boundary conditions, J. Differential Equations $\mathbf{7 7}$ (1989), 369-378.

[13] G. Huisken, Asymptotic behavior for singularities of the mean curvature flow, J. Differential Geom. 31 (1990), 285-299.

[14] T. Ilmanen, Convergence of the Allen-Cahn equation to Brakke's motion by mean curvature, J. Differential Geom. 38 (1993), 417-461.

[15] K. Kasai and Y. Tonegawa, A general regularity theory for weak mean curvature flow, Calc. Var. Partial Differential Equations 50 (2014), 1-68.

[16] A. N. Koeller, Regularity of mean curvature flows with Neumann free boundary conditions, Calc. Var. Partial Differential Equations 43 (2012), 265-309.

[17] O. A. Ladyženskaja, V. A. Solonnikov, and N. N. Ural'ceva, Linear and quasilinear equations of parabolic type, Translated from the Russian by S. Smith. Translations of Mathematical Monographs, Vol. 23, American Mathematical Society, Providence, R.I., 1967.

[18] C. Liu, N. Sato and Y. Tonegawa, Two-phase flow problem coupled with mean curvature flow, Interfaces Free Bound. 14 (2012), 185-203.

[19] C. Liu and N. J. Walkington, An Eulerian description of fluids containing visco-hyperelastic particles, Arch. Ration. Mech. Anal. 159 (2001), 229-252.

[20] M. Mizuno and Y. Tonegawa, Convergence of the Allen-Cahn equation with Neumann boundary conditions, SIAM J. Math. Anal. 47 (2015), 1906-1932.

[21] A. Stahl, Convergence of solutions to the mean curvature flow with a Neumann boundary condition, Calc. Var. Partial Differential Equations 4 (1996), 421-441.

[22] A. Stahl, Regularity estimates for solutions to the mean curvature flow with a Neumann boundary condition, Calc. Var. Partial Differential Equations 4 (1996), 385-407.

[23] A. Stone, A boundary regularity theorem for mean curvature flow, J. Differential Geom. 44 (1996), 371-434.

[24] A. G. Stone, Singular and boundary behaviour in the mean curvature flow of hypersurfaces, PhD thesis, Stanford University, 1994.

[25] K. Takasao, Gradient estimates and existence of mean curvature flow with transport term, Differential Integral Equations 26 (2013), 141-154.

[26] Y. Tonegawa, A second derivative Hölder estimate for weak mean curvature flow, Adv. Calc. Var. 7 (2014), 91-138.

[27] G. Wheeler and V.-M. Wheeler, Mean curvature flow with free boundary outside a hypersphere, arXiv preprint arXiv:1405.7774, 2014.

[28] V.-M. Wheeler, Mean curvature flow of entire graphs in a half-space with a free boundary, J. Reine Angew. Math. 690 (2014), 115-131.

[29] V.-M. Wheeler, Non-parametric radially symmetric mean curvature flow with a free boundary, Math. Z. 276 (2014), 281-298.

[30] W. P. Ziemer, Weakly differentiable functions, Graduate Texts in Mathematics, vol. 120, SpringerVerlag, New York, 1989.

(Masashi Mizuno) Department of Mathematics, College of Science and Technology, NiHON UNIVERSITY, TOKYO 101-8308 JAPAN

E-mail address: mizuno@math.cst.nihon-u.ac.jp

(Keisuke Takasao) Graduate School of Mathematical Sciences, University of Tokyo, Komaba 3-8-1, Meguro-Ku, ToKyo 153-8914, JAPAN

E-mail address: takasao@ms.u-tokyo.ac.jp 